\title{
Metabolically Healthy Obesity (MHO)—New Research Directions for Personalised Medicine in Cardiovascular Prevention
}

\author{
Peter M. Nilsson ${ }^{1} \cdot J^{\prime}$ han Korduner ${ }^{1} \cdot$ Martin Magnusson ${ }^{1,2,3}$
}

Published online: 17 February 2020

(C) The Author(s) 2020

\begin{abstract}
Purpose of Review To discuss new findings on the heterogeneity of obesity and associated risks.

Recent Findings Obesity is a public health problem of immense importance on a global scale. However, epidemiological findings and clinical studies have revealed that obesity is a heterogeneous phenotype and that not all obese subjects run the same risk for complications. Current research has tried to describe so-called metabolically healthy obesity (MHO), defined by lack of risk factors included in the metabolic syndrome. These subjects will not escape long-term complications, but mortality risk is not increased. However, a new definition of MHO has recently been proposed, based on the lack of hospitalisation for somatic disease for decades in middle life. MHO subjects defined in this way are characterised by being "fat and fit" and also run a lower risk of long-term complications.
\end{abstract}

Summary If MHO could be better understood, this could contribute to a more diverse clinical approach to obesity based on personalised medicine.

Keywords Cardiovascular $\cdot$ Diabetes $\cdot$ Health $\cdot$ Metabolic healthy obesity $\cdot$ Microbiome $\cdot$ Protection

\section{Introduction}

Even if obesity is a well-known risk factor for many aspects of morbidity and mortality [1], as well as causing a high economic burden to society [2], not all obese subjects have the same increased risk, thus showing heterogeneity. In fact, there are examples indicating that obesity may be protective when chronic disease is already present, for example chronic obstructive pulmonary disease (COPD) or congestive heart failure (CHF), when the prognosis seems to be better in more overweight or obese subjects than in leaner ones [3]. This has been referred to as cardiac cachexia in patients with CHF, linked to a state of chronic inflammation that could

This article is part of the Topical Collection on Hypertension and Obesity

Peter M. Nilsson peter.nilsson@med.lu.se

1 Department of Clinical Sciences, Department of Internal Medicine, Skåne University Hospital, Lund University, Jan Waldenströms gata 15, 5th floor, S-20502 Malmö, Sweden

2 Department of Cardiology, Clinical Research Centre (CRC), Jan Waldenströms gata 35, S-20502 Malmö, Sweden

3 Wallenberg Centre for Molecular Medicine, Lund University, Malmö, Sweden impact on weight loss and the associated worse prognosis [4]. Even subjects without chronic disease in mid-life have a better prognosis if they stay weight-stable in mid-life as compared with weight increase or weight loss based on observational data [5].

Also in subjects without such chronic disease conditions the negative impact on health of overweight/obesity is not uniform and there are many examples in the clinical setting, or based on population studies, that not all obese subjects will develop for example elevated blood pressure or impaired glucose metabolism that would otherwise be expected.

\section{Does Metabolically Healthy Obesity (MHO) Exist or Not?}

This finding of heterogeneity of risk has provoked a debate whether a phenotype of metabolically healthy obesity (MHO) exists or not, based on a definition of lacking features, or variables, of the so-called metabolic syndrome or by the level of insulin resistance, mainly by using the homeostasis model assessment of insulin resistance, HOMA-IR [6••, 7]. Protagonists of this hypothesis have shown data that such $\mathrm{MHO}$ individuals exist in epidemiological studies, but critics have stated that this represents only a temporary state and that sooner or later the burden of risk factors will become more visible and that the 
cardiometabolic event risk will therefore increase [8•]. Thus, according to this view, MHO is at best a temporary state and will not last, ultimately transforming into a metabolically unhealthy status [9]. Recent meta-analyses, based on this traditional definition of $\mathrm{MHO}$, have summarised that indeed the long-term risk of cardiovascular disease (CVD) is increased in former MHO subjects, but not total mortality risk [10••]. A new updated metaanalysis on the potential role of MHO in modifying risk of cardiovascular disease, total cancer, and all-cause and cause-specific mortality is presently underway based on published studies up until December 2019 according to a published protocol [11].

Another disputed topic is what kind of reference group should be used to compare MHO subjects with for risk estimates and clinical characteristics. Should it be the general non-obese population or a more selected subgroup of metabolically healthy, non-obese subjects as well as normal-weight individuals with a metabolically healthy phenotype [12]? This question is still not settled. If a very healthy reference group is selected, most other groups will show increased risk, but if the general population is used as reference, the comparison more resembles the real-world experience.

\section{A New Definition of MHO Is Presented}

To overcome these theoretical and conceptual problems, we have recently proposed a new definition to be used for classifying MHO subjects, based on data from two population-based studies in Malmö, southern Sweden. In the first study from the Malmö Preventive Project (MPP), we defined MHO subjects as the ones escaping hospitalisation for several decades of their mid-life and not developing type 2 diabetes, i.e. no transition to diabetes [13]. This group of non-hospitalised, non-diabetic MHO subjects with advanced obesity (BMI $\geq 35 \mathrm{~kg} / \mathrm{m}^{2}$ ) could well have a history of different risk factors or prescribed drugs, but the argument is that these traits or risk factors did not reach a point when need of hospital care was necessary, i.e. avoidance of hospitalisation. Thus, this MHO group could be compared in cross-sectional analyses with other similarly obese subjects with a history of hospitalisation as well as with non-obese controls. The findings indicated that the MHO group was characterised by a non-sedentary behaviour and therefore more like "fat and fit" than the obese group with a history of hospitalisation [13].

In a second study, based on the same definition of nonhospitalisation for decades in mid-life, data from the prospective Malmö Diet Cancer Study (MDCS) was used [14••]. It was found that $\mathrm{MHO}$ subjects (BMI $\geq 30 \mathrm{~kg} / \mathrm{m}^{2}$ ) without any hospitalisation for somatic health care before baseline in 1992 to 1996 (with exclusion of hospitalisation due to trauma or normal deliveries) had a significantly lower incident risk of prospective CVD and mortality as compared with Metabolically Unhealthy Obese (MUO) subjects (e.g. nondiabetic subjects with BMI $>30 \mathrm{~kg} / \mathrm{m}^{2}$ that had been hospitalised). In fact, the prospective risk of MHO subjects was similar to the one of non-obese controls, both metabolically healthy and unhealthy non-obese subjects [14••]. We are now going to further explore the genetic and biomarker characteristics of this non-hospitalised MHO group in a wellcharacterised sub-group of MDCS, the so-called Cardiovascular Cohort (MDCS-CC) [15]. Admittedly the $\mathrm{MHO}$ group is not without risk factors or metabolic abnormalities, and thus strictly speaking not completely metabolically healthy, but these adverse factors did not lead to severe disease causing need of hospitalisation until a mean of 56 years at MDCS baseline. Thus, it is more important to us to focus on the consequences of obesity (hospitalisation) than the levels of ever changing risk factors or prescribed drugs, many of these with infrequent use due to suboptimal compliance.

\section{Other Examples of Unexpected Risk Protection}

Why is it of importance to find out more about a subgroup of MHO individuals with a better prognosis than expected? This is not only because we need to increase knowledge about the heterogeneity of obesity in the population, or to develop a more diverse treatment strategy based on personalised medicine to offer people with obesity, but also in order to explore if specific protective mechanisms can be found or not. This is a strategy that in the end could eventually bring new drug targets if such protective mechanisms (if any) could be mapped, or if genetic analyses could reveal loss-of-function (LoF) mutations of harmful genes, as for PCSK-9 that lead to new drug development [16], but instead linked to obesity and cardiovascular risk. Such models of "extremes" can also be found in other areas of cardiovascular medicine, for example in subjects with the Supernormal Vascular Aging (SUPERNOVA) phenotype, as recently described [17], or in patients with type 1 diabetes of long duration but escaping major complications from the cardiovascular system, the kidneys or retinopathy $[18,19]$.

Still, we have reason to believe that MHO subjects are not fully protected from complications. Sooner or later such cardiovascular complications will develop also in the MHO subjects defined by our new definition (non-hospitalisation for somatic disease) [13, 14.•], but most likely such complications may be delayed for a shorter or longer time during the life course. This could bring benefits to the individual, but also lessen the burden on the health care system.

\section{Potential Mechanisms to Explain MHO}

Which mechanisms could possible explain the existence of $\mathrm{MHO}$ or the protection from cardiometabolic complications in subjects with obesity (Table 1)? One possibility is that no 
Table 1 Potential mechanisms contributing to metabolically healthy obesity (MHO), targets for further research

- Genetic variants, loss-of-function (LoF) mutations

- Reduced chronic inflammation

- Changes in adipose tissue composition

- Variants of adipocyte function

- Protection from non-alcohol fatty liver disease (NAFLD)

- Gastro-intestinal microbiota variation

- Less sedentary life-style ("fat and fit")

- Early life programming?

protective mechanisms exist at all, but that random variation could explain most of the findings. An alternative explanation could be that genetic factors involved in the regulation of hepatic function or glucose metabolism could be of importance. One indication of this is that not all obese subjects develop nonalcohol fatty liver disease (NAFLD), as an expected and very common negative consequence of obesity and insulin resistance [20]. As chronic inflammation is another well-known characteristic of obesity, and also involved in the development of cardiometabolic complications [21], it could be hypothesised that in true MHO subjects, inflammation is downregulated. This could be due to genetic factors, less pronounced NAFLD, or eventually a more benign and diverse microbiome of the bacterial content of the gastro-intestinal tract [22]. Future studies should try to elucidate further on the role of chronic inflammation, as now a recent intervention treatment study has shown that the reduction of inflammation by use of very specific mono-clonal antibody therapy (canakinumab against Interleukin 1-beta, IL-beta) was associated with a reduction of cardiovascular risk and events in the randomised, placebocontrolled Canakinumab Anti-Inflammatory Thrombosis Outcomes Study (CANTOS) trial [23].

\section{The Heart in Obesity_Unresolved Questions}

The link between obesity and cardiovascular changes leading to increased CVD morbidity and mortality is well established (both myocardial infarction and ischemic stroke), mediated by metabolic and vascular pathophysiological traits as well as other mechanisms. Even though it has been shown in in some, but not all, epidemiological studies that weight reduction is associated with decreased CVD risk, so far the results of studies on the effects of weight-reducing drugs, with the aim of reducing cardiovascular morbidity and mortality, have been disappointing [24]. One potential explanation for this is that the CVD risk associated with obesity is not causally related to overweight but instead to some other factor which is increased in parallel, for example insulin resistance or chronic inflammation. So, whereas it is clear that obese patients are at increased risk of hypertension, CVD, and heart failure, identification of new drug-modifiable biological pathways causally related to both obesity and CVD still remains challenging. Recently, the GLP-1 agonists have revealed a lot of attention due to their favorable effect in reducing cardiovascular risk in high-risk diabetes patients [25, 26]. Existing on the market to date for the treatment of obesity is the GLP-1 agonist liraglutide, but other similar drugs are also being tested, for example semaglutide. Its efficacy is comparable with other available agents and greater than that seen with orlistat or lorcaserin, but slightly less than weight loss seen with phentermine/topiramate combination treatment. However, liraglutide offers the unique benefit of improved glycemic control in addition to weight loss. Although it has been shown that liraglutide, apart from lowering weight and blood glucose in subjects with diabetes at high cardiovascular risk, also reduces mortality and CVD risk [26], additional studies are needed to determine its long-term efficacy and safety profile in non-diabetic obese patients [27].

Even if drug treatment still is under development to address obesity and its complication, the role of bariatric surgery is better established for long-term benefits, also for improving cardiac structure and function [28]. Such surgical interventions could also be considered for $\mathrm{MHO}$ subjects to relieve from symptoms of musculoskeletal and joint pain, or for improving self-esteem and reduce psycho-social stigma.

\section{Less Risk of Hypertension than Expected-Why?}

In the area of hypertension, there are also examples of subjects that have lower than expected blood pressure levels, for example in Iraqi immigrants to Malmö, Sweden, as compared with native, Caucasian Swedes [29]. Even if other features of the metabolic syndrome, such as increased waist circumference and dyslipidemia, were more pronounced in the Iraqis than in the Swedes, the blood pressure elevation was not in relative terms. This could be due to protective factors such as a better renal function [30], as shaped by evolutionary selection in subjects exposed to a very hot climate when the preservation of body fluids and keeping the electrolyte balance is of utmost importance. In a similar way, it was reported that Pima Indians also have somewhat lower mean blood pressure as compared with Caucasians, and also a lower sympathetic nervous activation [31], another factor that could impact on blood pressure regulation, but also on obesity when less energy is spent. Further studies are needed to disentangle biological factors from the socio-cultural factors that may apply during a migration process when lifestyle changes may occur.

\section{Future Research Directions}

If a more benign category of obesity could be defined, this would be a step forward in a similar way that, based on a new 
proposal for definition of diabetes, milder forms of diabetes could be defined with a less harmful course according to risk of complications [32•].

For understanding MHO, we need both observational studies on obesity and risk in different populations and ethnicities, but also basic science when characteristics of adipocytes from MHO subjects as well as the cardiovascular phenotype, including blood pressure regulation, should be investigated. Increased knowledge of genetic traits could bring insights into protective variants, for example loss-of-function mutations of harmful variants. In addition, the study of downregulated chronic inflammation could reveal protective patterns, possible to study by use of proteomics [33]. Finally, a better understanding of the role of GI microbiota and its interaction with dietary patterns could provide new perspectives. Also the role of the "fat and fit" phenotype, with corresponding personality traits should be more studied, as this could modify the current simplified message of weight loss in all obese subjects in a uniform way. In fact, to keep weight stable in middle age without the annually expected weight increase of $0.5-1.0 \mathrm{~kg} /$ year, as seen in many people, could bring a new message to some overweight/obese people, i.e. that keeping yourself weight-stable and fit is better than to be lean and unfit [34]. Lessons could also be learned from the Japanese sumo wrestlers that stay fat and fit during their sports career even if the high degree of obesity will increase risk factors [35], but after their career in many cases undergo a period of de-training and de-eating to avoid or lessen obesity complications.

\section{Conclusions}

The ongoing debate on whether MHO exists or not is important to bring new understanding and a more realistic clinical view on obesity. Most likely, the obesity phenotype is more heterogeneous than previously thought, similar to the modern view on the varying subtypes of diabetes carrying different risk of complications [32•]. If protective mechanisms associated with $\mathrm{MHO}$ could be identified, new treatment targets for new interventions (drugs, diet) could be defined. More direct research aims involve characterisation of adipocytes and cardiovascular function of MHO subjects, as well as the role of chronic inflammation, hepatic function, and GI microbiota to understand this condition [6••]. In the end, even if people with MHO could be identified, most likely the majority of them will neither escape cardiovascular complications nor diabetes in the long run, but if disease onset could be postponed by a decade or more, this could mean great benefits both for the individual and the health care system.

Funding Information Open access funding provided by Lund University. This review was financially supported by a grant from the Research
Council of Sweden, and from the Scania University Hospital funds to PMN.

\section{Compliance with Ethical Standards}

Conflict of Interest The authors declare no conflicts of interest relevant to this manuscript.

Human and Animal Rights and Informed Consent This article does not contain any studies with human or animal subjects performed by any of the authors.

Open Access This article is licensed under a Creative Commons Attribution 4.0 International License, which permits use, sharing, adaptation, distribution and reproduction in any medium or format, as long as you give appropriate credit to the original author(s) and the source, provide a link to the Creative Commons licence, and indicate if changes were made. The images or other third party material in this article are included in the article's Creative Commons licence, unless indicated otherwise in a credit line to the material. If material is not included in the article's Creative Commons licence and your intended use is not permitted by statutory regulation or exceeds the permitted use, you will need to obtain permission directly from the copyright holder. To view a copy of this licence, visit http://creativecommons.org/licenses/by/4.0/.

\section{References}

Papers of particular interest, published recently, have been highlighted as:

- Of importance

•• Of major importance

1. Di Angelantonio E, Bhupathiraju Sh N, Wormser D, Gao P, Kaptoge S, Global BMIMC, et al. Body-mass index and all-cause mortality: individual-participant-data meta-analysis of 239 prospective studies in four continents. Lancet. 2016;388(10046):776-86.

2. Tremmel M, Gerdtham UG, Nilsson PM, Saha S. Economic burden of obesity: a systematic literature review. Int J Environ Res Public Health. 2017;14(4).

3. Elagizi A, Kachur S, Lavie CJ, Carbone S, Pandey A, Ortega FB, et al. An overview and update on obesity and the obesity paradox in cardiovascular diseases. Prog Cardiovasc Dis. 2018;61(2):142-50.

4. Saitoh M, Ishida J, Doehner W, von Haehling S, Anker MS, Coats AJS, et al. Sarcopenia, cachexia, and muscle performance in heart failure: review update 2016. Int J Cardiol. 2017;238:5-11.

5. Nilsson PM, Nilsson JA, Hedblad B, Berglund G, Lindgarde F. The enigma of increased non-cancer mortality after weight loss in healthy men who are overweight or obese. J Intern Med. 2002;252(1):70-8.

6.• Smith GI, Mittendorfer B, Klein S. Metabolically healthy obesity: facts and fantasies. J Clin Invest. 2019;129(10):3978-89 This is a very thoughtful updated review with a research agenda to map mechanisms of potential importance for MHO. These authors describe a number of potential mechanisms linked to MHO that should be further explored, for example the role of adipocytes, inflammation, microbiota and non-alcohol fatty liver disease (NAFLD). If mechanisms could be better understood, this could lead to development of new drug targets for obese subjects. 
7. Kramer CK, Zinman B, Retnakaran R. Are metabolically healthy overweight and obesity benign conditions?: a systematic review and meta-analysis. Ann Intern Med. 2013;159(11):758-69.

8. Eckel N, Meidtner K, Kalle-Uhlmann T, Stefan N, Schulze MB. Metabolically healthy obesity and cardiovascular events: a systematic review and meta-analysis. Eur J Prev Cardiol. 2016;23(9):95666 In all 28 studies contributed to the meta-analysis. None of the approaches to define MHO clearly identified an obese subgroup not at increased risk of cardiovascular events compared with normal-weight healthy participants.

9. Eckel N, Li Y, Kuxhaus O, Stefan N, Hu FB, Schulze MB. Transition from metabolic healthy to unhealthy phenotypes and association with cardiovascular disease risk across BMI categories in 90257 women (the Nurses' Health Study): 30 year follow-up from a prospective cohort study. Lancet Diabetes Endocrinol. 2018;6(9):714-24.

$10 . \bullet$ Zheng R, Zhou D, Zhu Y. The long-term prognosis of cardiovascular disease and all-cause mortality for metabolically healthy obesity: a systematic review and meta-analysis. J Epidemiol Community Health. 2016;70(10):1024-31 This is a thorough and interesting systematic review based on 22 studies to show increased longterm cardiovascular risk, but not all-cause mortality, associated with the MHO phenotype.

11. Tian S, Liu Y, Feng A, Lou K, Dong H. Metabolically healthy obesity and risk of cardiovascular disease, cancer, and all-cause and cause-specific mortality: a protocol for a systematic review and meta-analysis of prospective studies. BMJ Open. 2019;9(10): e032742.

12. Hinnouho GM, Czernichow S, Dugravot A, Batty GD, Kivimaki M, Singh-Manoux A. Metabolically healthy obesity and risk of mortality: does the definition of metabolic health matter? Diabetes Care. 2013;36(8):2294-300.

13. Tremmel M, Lyssenko V, Zöller B, Engstrom G, Magnusson M, Melander $\mathrm{O}$, et al. Characteristics and prognosis of metabolically healthy obesity (MHO) - the Malmo Preventive Project. Obes Med. 2018;11:6-12.

14.• Korduner J, Bachus E, Jujic A, Magnusson M, Nilsson PM. Metabolically healthy obesity (MHO) in the Malmö diet cancer study - epidemiology and prospective risks. Obes Res Clin Pract 2019;13(6):548-554. https://doi.org/10.1016/j.orcp.2019.10.002 In this epidemiological study, a new definition of MHO is presented based on non-hospitalisation for somatic disease during several decades of mid-life, and with a prognosis that is comparable with non-obese subjects from the same population during follow-up.

15. Hedblad B, Nilsson P, Janzon L, Berglund G. Relation between insulin resistance and carotid intima-media thickness and stenosis in non-diabetic subjects. Results from a cross-sectional study in Malmö, Sweden. Diabet Med. 2000;17(4):299-307.

16. Klein-Szanto AJP, Bassi DE. Keep recycling going: new approaches to reduce LDL-C. Biochem Pharmacol. 2019;164:336-41.

17. Laurent S, Boutouyrie P, Cunha PG, Lacolley P, Nilsson PM. Concept of extremes in vascular aging. Hypertension. 2019;74(2):218-28.

18. Bain SC, Gill GV, Dyer PH, Jones AF, Murphy M, Jones KE, et al. Characteristics of type 1 diabetes of over 50 years duration (the Golden Years Cohort). Diabet Med. 2003;20(10):808-11.

19. Adamsson Eryd S, Svensson AM, Franzén S, Eliasson B, Nilsson PM, Gudbjörnsdottir S. Risk of future microvascular and macrovascular disease in people with type 1 diabetes of very long duration: a national study with 10-year follow-up. Diabet Med. 2017;34(3):411-8.
20. Byrne CD, Targher G. NAFLD: a multisystem disease. J Hepatol. 2015;62(1 Suppl):S47-64.

21. Bao X, Borné Y, Johnson L, Muhammad IF, Persson M, Niu K, et al. Comparing the inflammatory profiles for incidence of diabetes mellitus and cardiovascular diseases: a prospective study exploring the 'common soil' hypothesis. Cardiovasc Diabetol. 2018;17(1):87.

22. Ottosson F, Brunkwall L, Ericson U, Nilsson PM, Almgren P, Fernandez C, et al. Connection between BMI-related plasma metabolite profile and gut microbiota. J Clin Endocrinol Metab. 2018;103(4):1491-501.

23. Ridker PM, Everett BM, Thuren T, MacFadyen JG, Chang WH, Ballantyne C, et al. Antiinflammatory therapy with canakinumab for atherosclerotic disease. N Engl J Med. 2017;377(12):1119-31.

24. James WP, Caterson ID, Coutinho W, Finer N, Van Gaal LF, Maggioni AP, et al. Effect of sibutramine on cardiovascular outcomes in overweight and obese subjects. N Engl J Med. 2010;363(10):905-17.

25. Marso SP, Bain SC, Consoli A, Eliaschewitz FG, Jódar E, Leiter LA, et al. Semaglutide and cardiovascular outcomes in patients with type 2 diabetes. N Engl J Med. 2016;375(19):1834-44.

26. Marso SP, Daniels GH, Brown-Frandsen K, Kristensen P, Mann JF, Nauck MA, et al. Liraglutide and cardiovascular outcomes in type 2 diabetes. N Engl J Med. 2016;375(4):311-22.

27. Mehta A, Marso SP, Neeland IJ. Liraglutide for weight management: a critical review of the evidence. Obes Sci Pract. 2017;3(1):3-14.

28. Aggarwal R, Harling L, Efthimiou E, Darzi A, Athanasiou T, Ashrafian $\mathrm{H}$. The effects of bariatric surgery on cardiac structure and function: a systematic review of cardiac imaging outcomes. Obes Surg. 2016;26(5):1030-40.

29. Bennet L, Nilsson PM. Country of birth modifies the associations of body mass and hemoglobin A1c with office blood pressure in Middle Eastern immigrants and native Swedes. J Hypertens. 2014;32(12):2362-70.

30. Nilsson C, Christensson A, Nilsson PM, Bennet L. Renal function and its association with blood pressure in Middle Eastern immigrants and native Swedes. J Hypertens. 2017;35(12):2493-500.

31. Weyer C, Pratley RE, Snitker S, Spraul M, Ravussin E, Tataranni PA. Ethnic differences in insulinemia and sympathetic tone as links between obesity and blood pressure. Hypertension. 2000;36(4):531-7.

32. Ahlqvist E, Storm P, Käräjämäki A, Martinell M, Dorkhan M, Carlsson A, et al. Novel subgroups of adult-onset diabetes and their association with outcomes: a data-driven cluster analysis of six variables. Lancet Diabetes Endocrinol. 2018;6(5):361-9 Here a new definition of diabetes is proposed based on five subtypes and linked to varying risk, thus showing the heterogeneity of diabetes and risk that could also be applicable to obesity.

33. Benabdelkamel H, Masood A, Okla M, Al-Naami MY, Alfadda AA. A proteomics-based approach reveals differential regulation of urine proteins between metabolically healthy and unhealthy obese patients. Int J Mol Sci. 2019;20(19).

34. Lee CD, Blair SN, Jackson AS. Cardiorespiratory fitness, body composition, and all-cause and cardiovascular disease mortality in men. Am J Clin Nutr. 1999;69(3):373-80.

35. Nishizawa T, Akaoka I, Nishida Y, Kawaguchi Y, Hayashi E. Some factors related to obesity in the Japanese sumo wrestler. Am J Clin Nutr. 1976;29(10):1167-74.

Publisher's Note Springer Nature remains neutral with regard to jurisdictional claims in published maps and institutional affiliations. 